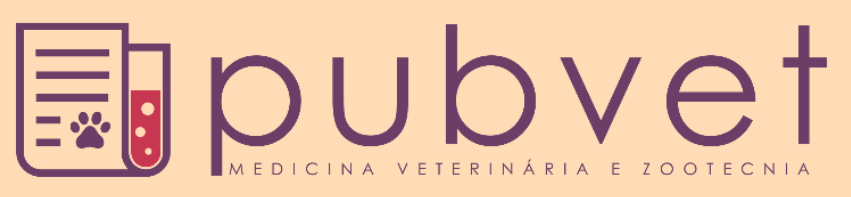

https://doi.org/10.31533/pubvet.v15n07a875.1-7

\title{
Tomada de decisão quanto ao uso de medidas profiláticas na produção de animais melhoradores
}

\author{
Éverton Mrás da $\operatorname{Paz}^{1 \oplus}$, Felipe Anicet Bittencourt ${ }^{2}{ }^{\bullet}$, Gabriela Maia de Azevedo $^{3}{ }^{\bullet}$, Verônica \\ Schmidt $^{4 * 0}$ (D)
}

${ }^{I}$ Dielat - Indústria e Comércio de Laticínios, Taquara, RS.

${ }^{2}$ Zootecnista autônomo, Dom Pedrito, $R S$.

${ }^{3}$ Programa de Pós-graduação em Zootecnia, UFPEL, Pelotas, RS,

${ }^{4}$ Professor Titular - Departamento de Medicina Veterinária Preventiva/UFRGS, Porto Alegre, RS.

*Autor para correspondência, E-mail: veronica.schmidt@ufrgs.br

Resumo. O desempenho de um sistema de produção necessita constantemente de boas práticas sanitárias, minimizando riscos do desenvolvimento de doenças nos rebanhos, por meio de medidas profiláticas preventivas ou curativas, bem como a adoção de medidas de higiene. Neste sentido, realizou-se um estudo analítico-descritivo sobre as ações realizadas por produtores rurais após a participação em uma exposição internacional de animais, no Estado do Rio Grande do Sul, com o objetivo de identificar as medidas profiláticas adotadas quando no retorno às propriedades de origem. Aplicaram-se questionários semiestruturados, na forma de entrevista, a 13 produtores rurais, os quais trabalham com diferentes espécies e raças, amostrados intencionalmente e tendo como critério de seleção o interesse e disponibilidade em participar da pesquisa. Nas entrevistas, os produtores foram questionados sobre o manejo estabelecido em relação às medidas de higiene e profilaxia dos animais que participam de exposições e feiras, bem como sobre a incidência de doenças no rebanho quando no retorno à unidade produtiva (UP). Dos treze entrevistados, três (23\%) relataram adotar medidas de manejo diferenciado no retorno dos animais à propriedade. A ocorrência de enfermidades nos animais após o retorno à UP foi relatada em três UPs. O resultado encontrado foi diferente do esperado ( $\mathrm{P}<0,001$ ), supondo que a não adoção de manejo sanitário adequado possa culminar com aumento na incidência de agravos à saúde. Embora a baixa incidência de doenças nos rebanhos seja relatada pelos entrevistados, as medidas de higiene e profilaxia são ferramentas imprescindíveis à prevenção e controle de enfermidades transmissíveis.

Palavras-chave: Higiene, profilaxia, manejo preventivo

\section{Decision-making on prophylactic measures in animal breeding farms}

\begin{abstract}
The performance of a production system constantly requires good sanitary practices, minimizing the risks of disease development in the herds, through preventive or curative prophylactic measures, as well as the adoption of hygiene measures. In this sense, an analytical-descriptive study was carried out on the actions taken by rural producers after participating in an international exhibition of animals, in the State of Rio Grande do Sul, in order to identify the prophylactic measures adopted when returning to the properties source. Semi-structured questionnaires were applied, in the form of interviews, to 13 rural producers, who work with different species and races, intentionally sampled and having as selection criteria the interest in participating in the research. The producers were asked about the management established in relation to the hygiene and prophylaxis measures with the animals participating in exhibitions and fairs, as well as on the incidence of diseases in the herd when returning to the production unit. Of the thirteen interviewees, three (23\%)
\end{abstract}


reported adopting differential management measures on the return of animals to property; the occurrence of diseases in the animals after the return to the UP, was reported in three UPs. The result found was significantly different from the expected one $(\mathrm{P}<0.001)$, if not adopting adequate health management could culminate with an increase in the incidence of diseases Cheers. Although the low incidence of diseases in the herds is reported by the interviewees, hygiene and prophylaxis measures are essential tools for the prevention and control of communicable diseases.

Keywords: Hygiene, prophylaxis, preventive management

\section{Tomada de decisiones sobre el uso de medidas profilácticas en la producción de animales de exposición}

Resumen. El desempeño de un sistema de producción requiere constantemente buenas prácticas sanitarias, minimizando los riesgos de desarrollo de enfermedades en los rebaños, mediante medidas profilácticas preventivas o curativas, así como la adopción de medidas de higiene. En este sentido, se realizó un estudio analítico-descriptivo sobre las acciones realizadas por parte de los productores rurales después de participar en una exhibición internacional de animales, en el Estado de Rio Grande do Sul, con el objetivo de identificar las medidas profilácticas adoptadas al regresar a las propiedades. Se aplicaron cuestionarios semiestructurados, en forma de entrevista, a 13 productores rurales, que trabajan con diferentes especies y razas, muestreados intencionalmente y teniendo como criterio de selección el interés y disponibilidad para participar en la investigación. En las entrevistas se preguntó a los productores sobre el manejo establecido en relación con las medidas de higiene y profilaxis con los animales que participan en exposiciones y ferias, así como sobre la incidencia de enfermedades en el rebaño al regresar a la unidad de producción (UP). De los trece encuestados, tres (23\%) informaron haber adoptado diferentes medidas de manejo al devolver animales a la propiedad; la ocurrencia de enfermedades en animales luego de regresar a UP, fue reportada en tres UP. El resultado encontrado fue diferente al esperado $(\mathrm{P}<0,001)$, asumiendo que la falta de adopción de un manejo sanitario adecuado puede culminar en un aumento de la incidencia de problemas de salud. Si bien los entrevistados informan sobre la baja incidencia de enfermedades en los rebaños, las medidas de higiene y profilaxis son herramientas fundamentales para la prevención y control de las enfermedades transmisibles.

Palabras clave: Higiene, profilaxia, manejo preventivo

\section{Introdução}

Estamos na era das doenças emergentes e reemergentes, a maioria zoonóticas, e vários fatores são responsáveis pela situação atual, entre estes a movimentação de animais e o desenvolvimento socioeconômico de determinadas regiões (Megid et al., 2016). Tal fato enfatiza a importância da aplicação de medidas de higiene e profilaxia, especialmente no que diz respeito à produção animal, com intuito mais abrangente, mas não somente, de garantir a integridade da saúde humana.

As avaliações econômicas são partes integrantes de estudos epidemiológico pois justificam ações do setor público em sanidade animal, as medidas de controle e barreiras. Isto porque, uma doença em sistemas produtivos reduz a quantidade e/ou qualidade de produtos ao consumo humano, aumentando os custos de produção. Neste sentido, a ocorrência de doenças multifatoriais envolve os vários determinantes associados ao hospedeiro, agente e meio ambiente e, por este motivo, as previsões realizadas no domínio da exploração e da saúde animal revestem-se de numerosos fatores de incerteza (Thrusfield, 2004).

O desempenho de um sistema produtivo necessita da adoção de boas práticas agropecuárias, incluindo a utilização de manejo sanitário profilático e práticas de higiene. Desta forma, é possibilitado aos animais expressarem seus potenciais genéticos e, consequentemente, melhorarem os resultados de produtividade nos empreendimentos. Não obstante, adotando medidas corretas de manejo sanitário na 
produção e criação animal, além da promoção de bem-estar animal, são mitigados impactos ambientais negativos como, por exemplo, a redução do uso de medicamentos curativos.

Em exposições agropecuárias os animais possuem alto valor zootécnico e são comercializados como matrizes e reprodutores melhoradores assim, o objetivo do presente estudo foi identificar as medidas profiláticas adotadas por expositores com os animais expostos em uma feira agropecuária, quando do retorno às propriedades de origem.

\section{Metodologia}

Realizou-se um estudo descritivo e analítico com expositores de animais em uma feira agropecuária internacional, no Rio Grande do Sul, com o objetivo de identificar as medidas profiláticas adotadas, quando no retorno às propriedades de origem. Aplicaram-se questionários semiestruturados, na forma de entrevista, a 13 produtores rurais, os quais trabalham com diferentes espécies e raças. A amostragem foi intencional, tendo como critério de seleção o interesse e disponibilidade do produtor em participar do estudo.

A exigência estabelecida na Resolução 196/96 do Conselho Nacional de Saúde (Brasil, 1996) de que toda pesquisa que envolva humanos se processe após consentimento livre e esclarecido dos sujeitos, indivíduos ou grupos foi atendida à medida que, ao responder o questionário, a pessoa concordou com participação na pesquisa.

Nas entrevistas, os produtores foram questionados sobre o manejo estabelecido em relação às medidas de higiene e profilaxia com os animais que participam de exposições e feiras, bem como sobre a incidência de doenças no rebanho quando no retorno à unidade produtiva (UP). A análise dos dados foi realizada pelo teste de Chi-quadrado e determinação do índice Kappa, com auxílio do software GraphPad, em nível de 5\% de significância.

\section{Resultados e discussão}

De acordo com Silva Júnior et al. (2017), o comércio de animais é um importante fator de risco para a disseminação de doenças entre animais e rebanhos e as feiras desempenharam um papel importante nesta rede. Isto por que, no período de 2012 a 2013 em Pernambuco, 52\% das movimentações de bovinos envolveu entrada ou saída de feiras de gado, representando cerca de dois milhões de cabeças. $\mathrm{Na}$ Exposição Internacional de Animais (Expointer), em Esteio, Rio Grande do Sul, são expostos cerca de quatro mil animais a cada ano.

Entre os entrevistados, apenas três (23\%) realizavam procedimento profilático com o rebanho profilaxia corresponde ao conjunto de medidas médicas e sanitárias que visam prevenir o aparecimento de uma doença, limitar o seu desenvolvimento e assegurar o seu desaparecimento (Toma et al., 2004), mantendo em quarentena os animais provenientes de exposições e feiras agropecuárias (EFAs). Também, Teixeira et al. (2015) verificaram que a realização de quarentena para os animais recémchegados na unidade produtiva foi uma das práticas adotadas em menor frequência nos rebanhos maranhenses de caprinos e ovinos (19,5\% e 14,5\%), respectivamente. De acordo com Thrusfield (2004), a quarentena é o isolamento de animais, tanto infectados quanto suspeitos, assim como animais não infectados, mas em risco. Trata-se de um método antigo de controle que ainda é muito valioso. Ainda, segundo o mesmo autor, as medidas profiláticas podem ser adotadas conforme os níveis de prevenção (primária: impedir a introdução das doenças nos sistemas produtivos; secundária: ações de prevenção dirigidas às fontes de infecção, que incluem sua identificação e intervenção o mais rapidamente possível por meio do sacrifício, isolamento ou tratamento; terciária: medidas são aplicadas às consequências das doenças). Desta forma, genericamente, as medidas de profilaxia podem ser agrupadas em: medidas de prevenção, controle e erradicação. As medidas profiláticas sanitárias podem ser categorizadas como medidas ofensivas e defensivas. As medidas defensivas têm por objetivo proteger uma exploração indene e impedir ou dificultar a introdução de agentes patogênicos nesta. Por outro lado, a introdução de animais suscetíveis - principal risco sanitário - em unidades produtivas com presença do patógeno, mas com controle da doença, leva à ocorrência de manifestação clínica da enfermidade. Neste sentido, o alojamento de animais em um local de quarentena (em geral uma quinzena após a chegada à UP), enquanto aguardam a realização de testes diagnósticos (Toma et al., 2004) é recomendado. 
De acordo com (Silva Junior \& Luvizotto, 2013), no Brasil, o agronegócio é caracterizado por grande heterogeneidade entre os vários tipos de UPs; porém, a forma de produção que se destaca no país é a pequena propriedade de base familiar. Entretanto, esta não é a realidade absoluta observada entre produtores de animais que participam de exposições agropecuárias, onde os animais, em princípio, possuem alto valor zootécnico e potencial genético melhorador, validados por registro genealógico. Este é um fator que contribui para o aumento no valor econômico destes animais restringindo, assim, o número de produtores com investimentos para esta finalidade.

O processo decisório tem sido objeto de estudo de vários autores, resultando em abordagens diferenciadas, que vão desde as teorias clássicas da administração até áreas específicas da psicologia. Por isso, contempla aspectos políticos da decisão, aspectos comportamentais dos envolvidos no processo e, até mesmo, fatores como a intuição (Prado \& Souza, 2009). A escolha da melhor opção possível é a tomada de decisão (TD). Para Pereira et al. (2009) e Souza et al. (2015) esta decisão é difícil por causa do aumento da complexidade gerencial, ritmo das atividades e processos de mudanças.

A tomada de decisão (TD), especialmente a qualidade das decisões, tem impacto significativo no desempenho da organização e assume importância estratégica para o futuro desta. Assim, a TD não é um processo fácil, pois associada à cada alternativa de decisão está a incerteza de suas consequências e impactos. De fato, nem todas as decisões são complexas e demoradas e, embora as decisões estratégicas tenham maior visibilidade, são as pequenas decisões, tomadas rotineiramente, que permite à organização alcançar seus objetivos (Sobral \& Peci, 2008).

Dalcin et al. (2010) observaram que o pequeno produtor não responde a critérios simples de otimização. As decisões são baseadas, na maioria dos casos, no uso do bom senso e no conhecimento empírico e, por outro, na visão global de seu meio que o faz considerar um complexo de consequências de acordo com os objetivos que pretende atingir. Desta forma, passa a agir e a gerir seu sistema de produção conferindo-lhe uma lógica, uma racionalidade que lhe é própria, condicionada por um ambiente físico, social, cultural, institucional, político e econômico. Neste sentido, torná-lo conhecedor dos fatores extrínsecos à propriedade poderia proporcionar um melhor custo-benefício na produção (Prado \& Souza, 2009).

Na maior parte do tempo, a TD se faz escolhendo a opção mais rentável, ou seja, menos onerosa. Em geral, estudos econômicos em saúde são simples. Entretanto, a execução destes é dependente da incerteza da evolução da situação epidemiológica da doença, da aplicação das medidas escolhidas e de seu impacto sobre a doença. Em certas situações, a utilização de uma árvore de decisão pode clarear a situação e facilitar TD. Estes estudos, mesmo estando associados a um risco de erro irredutível, são indispensáveis antes de qualquer decisão sanitária (Toma et al., 2004).

Entre os dez produtores entrevistados que não adotam nenhuma medida profilática ao retorno de EFAs, apenas dois relataram a ocorrência de problemas sanitários no rebanho. O resultado encontrado foi significativamente diferente do esperado $(\mathrm{P}<0,001)$, supondo que a não adoção de manejo sanitário adequado pode culminar com aumento na incidência de agravos à saúde. Entretanto, no presente estudo, também a adoção de medidas profiláticas em animais participantes de EFAs, resultou em relatos de enfermidade nos lotes (Figura 1).

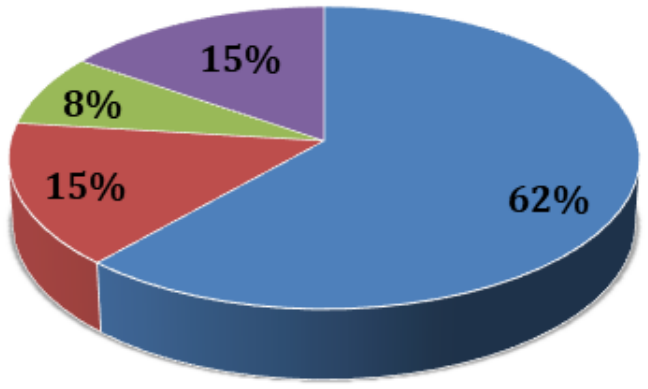

$$
\begin{aligned}
& \text { - sem manejo e sem problema } \\
& \text { - sem manejo e com problema } \\
& \text { = com manejo e com problema } \\
& \text { - com manejo e sem problema }
\end{aligned}
$$

Figura 1. Frequência (\%) de unidades produtivas com e sem problema sanitário após utilização ou não de manejo profilático em animais participantes de exposição agropecuária. 
O elevado percentual de UPs que não adotam medidas profiláticas nas quais não foram relatados problemas sanitários, resultou em discordância entre estas duas medidas $(\mathrm{k}=-0,073)$. Isto pode ser decorrente da capacidade de resistência destes animais, uma vez que eles se encontram preparados para a exposição. Sem uma interferência humana mais constante, os animais tendem a ser selecionados naturalmente para características como rusticidade e resistência às doenças e parasitas. $\mathrm{O}$ desenvolvimento da resistência pode ser direcionado por meio de cruzamentos entre raças, pelo estímulo gradual do sistema imune, ou mesmo na ingestão do colostro pelos animais recém-nascidos. Tizard (2014) reforça que a presença de imunoglobulinas no sangue é determinante para prevenir o aparecimento de doenças, bem como no intestino, onde estas atuam localmente inviabilizando ou impedindo a aderência de microrganismos (Rodríguez et al., 2009).

Embora a baixa incidência de doenças nas UPs integrantes do presente estudo, as medidas de higiene e profilaxia permanecem mister para prevenção de surtos e epidemias. De um modo geral, seguindo o pensamento de Machado \& Miguel (2010), pode-se afirmar que em toda e qualquer atividade de produção o risco é uma constante. Entendendo-se por risco a possibilidade de ocorrência de alguma adversidade ou perda, na principal função dos administradores que é tomar decisões, enfrentar ou gerenciar riscos. Na avaliação de risco, os perigos são identificados e depois a probabilidade e magnitude do risco real e preditivo, associadas aos perigos são quantificados pela análise de dados históricos e elaboração de modelos (Thrusfield, 2004).

Há de se considerar que as enfermidades relatadas, dificilmente poderiam ter nos demais animais expostos sua fonte de infecção, por estes serem rigorosamente avaliados no ingresso às exposições, regidas por um regulamento interno, condizente com a Portaria n. ${ }^{\circ} 108 / 93$, de 17 de março de 1993, do Ministério da Agricultura (Brasil, 1993). No caso, a UP01 relatou a ocorrência dermatomicose. Esta enfermidade, segundo Avante et al. (2009), encontra no confinamento condições favoráveis para sua propagação, que se dá pelo contato direto dos animais sadios com o ambiente infestado ou com aqueles animais de manifestação subclínica juntamente confinados. Neste sentido, a legislação vigente (Brasil, 1993) prevê, no capítulo que discorre sobre o controle sanitário das exposições, feiras, leilões e outras aglomerações de animais, que todos os animais deverão ser obrigatoriamente examinados por médico veterinário na entrada dos recintos, sendo admitidos quando não apresentarem sinais clínicos de doenças e estejam livres de parasitas externos, assim como acompanhados da documentação sanitária requerida segundo a espécie animal, regularmente expedida por médico veterinário no local de procedência.

Tristeza parasitária bovina foi relatada na UP04, no rebanho que foi exposto. Esta é uma doença comum transmitida por carrapato e causadora de prejuízos bilionários à pecuária do país anualmente (Almeida et al., 2006). Gonçalves (2000), em uma revisão sobre a enfermidade, aponta que as perdas econômicas são devido à redução na produção de leite e carne, infertilidade temporária de machos e fêmeas, custo de tratamentos, gasto com medidas preventivas necessárias, quando se introduz animais de áreas livres em áreas endêmicas e, principalmente, devido à mortalidade. Novamente, neste caso, deve-se atentar para a legislação vigente, no que dita a submissão dos animais ao julgamento de admissão ao parque.

A correta adoção das medidas de controle com cunho profilático, tais como a quarentena, visa assegurar também plenitude de bem-estar aos lotes de animais que retornam às UPs de origem (Feldman, 1997). Neste sentido, buscam compensar os fatores estressantes suportados durante o período determinado para exposição na feira, no qual o sistema imunológico dos animais foi desafiado repentinamente, desde a movimentação para mudança de ambiente até a troca da alimentação fornecida rotineiramente, como relatado pela UP12. De acordo com Stotzer et al. (2014), erros na alimentação facilmente conduzem ao aparecimento de problemas e uma alimentação correta pode mesmo "mascarar" outros erros de manejo, não sendo então estes aparentes. Do equilíbrio alimentar depende o equilíbrio celular, conhecido como homeostase. Um animal carenciado ou sobrealimentado apresenta proporcionalmente produções mais baixas, além de ser atreito a doenças. No mesmo sentido, os alimentos podem conter fatores exógenos indesejáveis, tais como microrganismos, resíduos químicos, entre outros, prejudiciais à saúde. Contudo, há de se considerar que o foco dos questionamentos foram os expositores sendo que os animais vendidos durante a feira, serão encaminhados a outras UPs e, sobre as quais, desconhece-se o manejo profilático adotado. 
A priori, não se pode saber se os focos de uma doença se desencadearão ou não nos próximos meses ou anos, mesmo tomando o máximo de precauções de proteção (Toma et al., 2004). Neste sentido, a maior compreensão dos fatores que condicionam o comportamento humano e os avanços tecnológicos têm permitido desenvolver um conjunto de ferramentas que melhoram significativamente a eficácia da tomada de decisão (Sobral \& Peci, 2008).

Com sabedoria e razoabilidade, percebemos os animais menos como máquinas biológicas e mais como seres vivos - intimamente relacionados ao meio ambiente. Tal percepção nos leva ao fato de que muitos problemas podem ser corrigidos pelas boas práticas de manejo sanitário, mitigando o impacto do uso de medicamentos curativos. A despeito do fato de as populações de animais de produção nacionais assumirem valores muito expressivos, os níveis de produção e de produtividade atingidos pelos rebanhos ainda estão muito aquém do que pode ser alcançado com a tecnologia disponível. Os fatores limitantes incluem aspectos nutricionais, sanitários, socioeconômicos e culturais. Assim, o grande desafio atual é aumentar, de maneira sustentável, a produtividade e a competitividade da pecuária brasileira.

Para tomar uma decisão os agricultores precisam conhecer o ambiente onde estão inseridos, as relações existentes entre as atividades que acontecem dentro das propriedades rurais com aquelas que ocorrem quando os produtos são destinados ao consumo e preservar os recursos naturais da propriedade.

Idealizando um modelo de manejo sanitário adequado, Kamwa (2010) enfatiza que o uso profilático de medicamentos contra patógenos em animais é um recurso ao qual se deve recorrer em circunstâncias de absoluta necessidade, respeitando-se a dosagem tipicamente preventiva. Esta cautela é necessária para não determinar o desenvolvimento de resistência aos fármacos pelos respectivos patógenos, bem como para garantir a inocuidade dos alimentos originados da exploração animal. Outra medida que pode ser adotada é a rotação de princípios ativos, aplicando-os em épocas, dosagens e situações corretas.

\section{Conclusão}

O estudo realizado demonstrou que há manejos diferentes em relação ao perfil dos produtores e que a cultura local pode ser um fator agravante ao mau gerenciamento dos riscos nas criações. Embora tenha sido referida baixa incidência de doenças nos rebanhos das unidades produtivas entrevistadas, as medidas de higiene e profilaxia permanecem mister para prevenção da ocorrência de agravos à saúde. Da mesma forma, a existência de registros é fundamental para previsão de problemas futuros com os rebanhos, direcionando as ações além do calendário de vacinação obrigatória, caso existente, somando esforços à fase atual da profilaxia - de vigilâncias e ações coletivas.

\section{Referências}

Almeida, M. B., Tortelli, F. P., Riet-Correa, B., Ferreira, J. L. M., Soares, M. P., Farias, N. A. R., RietCorrea, F., \& Schild, A. L. (2006). Tristeza parasitária bovina na região sul do Rio Grande do Sul: estudo retrospectivo de 1978-2005. Pesquisa Veterinária Brasileira, 26(4), 237-242.

Avante, M. L., Campos, C. P., Ferreira, M. M. G., Martins, I. S., Rosa, B. R. T., Sousa, G. D. P., \& Avanza, M. F. B. (2009). Dermatófitose em grandes animais. Revista Científica Eletrônica de Medicina Veterinária, 12.

Brasil. Conselho Nacional de Saúde. (1996). Resolução No 196/96. Estabelece as diretrizes e normas regulamentadoras de pesquisas envolvendo seres humanos. Disponível em: http://www.ufrgs.br/bioetica/res19696.htm.

Brasil. Ministério da Agricultura, Pecuária e Abastecimento (1993). Portaria n. ${ }^{\circledR}$ 108/93, de 17/03/93. Normas técnicas para organização e funcionamento das exposições e feiras agropecuárias, leilões rurais e dos Colégios de Jurados das associações encarregadas da execução dos serviços de registro genealógico. MAPA, Brasília, DF, Brasil. Disponível: http://www.indea.mt.gov.br/documents/363967/11673994/Portaria+108-Anexo.pdf/caf0725e19bd-1835-cfea-8dc82a162043

Dalcin, D., Oliveira, S. V., Troian, A., \& Lucena, L. P. (2010). O processo de tomada de decisão e a teoria da contingência no setor agrícola: um estudo de caso para o município de Boa Vista das 
Missões (RS). INTERNET.

Feldman, E. C. (1997). Tratado de medicina interna veterinária. In Moléstias do cão e do gato (Vol. 3).

Gonçalves, P. M. (2000). Epidemiologia e controle da tristeza parasitária bovina na região sudeste do Brasil. Ciência Rural, 30(1), 187-194. https://doi.org/10.1590/s0103-84782000000100030.

Kamwa, E. B. (2010). Biosseguridade, higiene e profilaxia: abordagem teórico-didática e aplicada. Nandyala.

Machado, J. A. D., \& Miguel, L. A. (2010). Elementos do processo de tomada de decisão. In S. A. Wagner, E. Giasson, L. A. Miguel, \& J. A. D. Machado (Eds.), Gestão de planejamento de unidade de produção agrícola. Universidade Federal do Rio Grande do Sul, Faculdade de Veterinária.

Megid, J., Ribeiro, M. G., \& Paes, A. C. (2016). Doenças infecciosas em animais de produção e de companhia. Roca.

Pereira, L. B., Souza, J. P., \& Cário, S. A. F. (2009). Elementos básicos para estudo de cadeias produtivas: tratamento teórico-analítico. In I. N. 'Prado \& J. P. Souza (Eds.), Cadeias produtivas: estudos sobre competitividade e coordenação (pp. 1-38). Eduem.

Prado, I. N., \& Souza, J. P. (2009). Cadeias produtivas: estudo sobre a competitividade e coordenação (Vol. 1). Eduem.

Rodríguez, C., Castro, N., Capote, J., Morales-delaNuez, A., Moreno-Indias, I., Sánchez-Macías, D., \& Argüello, A. (2009). Effect of colostrum immunoglobulin concentration on immunity in Majorera goat kids. Journal of Dairy Science, 92(4), 1696-1701. https://doi.org/http://dx.doi.org/10.3168/jds.2008-1586

Silva Junior, J. G., \& Luvizotto, C. K. (2013). Sustentabilidade do agronegócio: um panorama sociológico. Colloquium Humanarum, $10, \quad$ 585-593. https://doi.org/10.5747/ch.2013.v10.nesp.000500.

Silva Júnior, J. L., Almeida, E. C., Corrêa, F. N., Lima, P. R. B., Ossada, R., Marques, F. S., Dias, R. A., Ferreira, F., Neto, J. S. F., \& Grisi-Filho, J. H. H. (2017). Livestock markets play an important role in the cattle movement network in Pernambuco, Brazil. Brazilian Journal of Veterinary Research and Animal Science, 54(3), 225-237. https://doi.org/10.11606/issn.16784456.bjvras.2017.124303.

Sobral, F., \& Peci, A. (2008). Administração: teoria e prática no contexto brasileiro. Pearson Prentice Hall.

Souza, Â. R. L., Machado, J. A. D., \& Dalcin, D. (2015). Análise de estudos internacionais sobre os fatores que influenciam a decisão dos agricultores pela produção orgânica. Revista Em Agronegócio e Meio Ambiente, 8(3), 563-583. https://doi.org/10.17765/2176-9168.

Stotzer, E. S., Lopes, L. B., Eckstein, C., de Moraes, M. C. M. M., Rodrigues, D. S., \& Bastianetto, E. (2014). Impacto econômico das doenças parasitárias na pecuária. Revista Brasileira de Higiene e Sanidade Animal, 8(3), 198-221.

Teixeira, W. C., Santos, H. P., Silva, J. C. R., Rizzo, H., Marvulo, M. F. V., \& Castro, R. S. (2015). Perfil zoosanitário dos rebanhos caprinos e ovinos em três mesorregiões do estado do Maranhão, Brasil. Acta Veterinaria Brasilica, 9(1), 34-42. https://doi.org/10.21708/avb.2015.9.1.4438.

Thrusfield, M. V. (2004). Epidemiologia Veterinária (Vol. 1). Roca, Brasil.

Tizard, I. R. (2014). Imunologia Veterinária: introdução. Editora Roca.

Toma, B., Dufour, B., Sanaa, M., Benet, J.-J., Shaw, A., Moutou, F., \& Louzã, A. (2004). Epidemiologia aplicada: à luta colectiva contra as principais doenças animais transmissíveis. Fundação Calouste Gulbenkian.

Histórico do artigo:

Recebido: 21 de janeiro de 2021

Aprovado: 22 de março de 2021
Licenciamento: Este artigo é publicado na modalidade Acesso Aberto sob a licença Creative Commons Atribuição 4.0 (CC-BY 4.0), a qual permite uso irrestrito, distribuição, reprodução em qualquer meio, desde que o autor e a fonte sejam devidamente creditados. 